\title{
BMJ Open Quality Ensuring adequate vascular access in patients with major trauma: a quality improvement initiative
}

\author{
Kevin Verhoeff, ${ }^{1}$ Rachelle Saybel, ${ }^{2}$ Pamela Mathura, ${ }^{3}$ Bonnie Tsang, ${ }^{2}$ \\ Vanessa Fawcett, ${ }^{2}$ Sandy Widder ${ }^{2}$
}

To cite: Verhoeff K, Saybel R, Mathura $\mathrm{P}$, et al. Ensuring adequate vascular access in patients with major trauma: a quality improvement initiative.BMJ Open Quality 2018;7:e000090. doi:10.1136/ bmjoq-2017-000090

Received 19 April 2017 Revised 29 November 2017 Accepted 8 December 2017

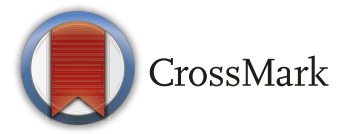

${ }^{1}$ Faculty of Medicine and Dentistry, University of Alberta, Edmonton, Alberta, Canada ${ }^{2}$ Department of Surgery, University of Alberta, Edmonton, Alberta, Canada

${ }^{3}$ Department of Medicine, University of Alberta, Edmonton, Alberta, Canada

Correspondence to Dr Sandy Widder; Sandy.Widder2@albertahealth services.ca

\section{ABSTRACT}

Ensuring adequate vascular access in major trauma patients prior to decompensative physiological processes is crucial to patient outcomes. Most protocols suggest achieving two 18-gauge or larger intravenous lines immediately in patients with major trauma. We discuss a quality improvement approach to ensure that $>90 \%$ of patients with major trauma (as defined by an injury severity score $\geq 12$ ) at a level one trauma centre receive timely and adequate fluid access. Applying Donabedian principles for process improvement, we used the Alberta Trauma Registry to perform a 4-month chart audit on patients with major trauma at the University of Alberta Hospital. Background data were supported with a formal root cause analysis to outline the problems and generate plan, do, study and act (PDSA) rapid change cycles. These PDSA cycles were then implemented over the course of 2 months to alter system and personnel barriers to care, thereby ensuring that patients with major trauma received adequate vascular access for fluid resuscitation. This was followed by a 6 -month sustainability assessment. The percentage of patients with major trauma who received adequate fluid access went from a mean of $55.5 \%$ to $>90 \%$ in 2 months and was sustained at or greater than $90 \%$ for 6 consecutive months. The formal application of quality improvement processes is uncommon in trauma care but is much needed to ensure success and sustainability of quality initiatives. Planning including engagement and prechange awareness is crucial to staff engagement, change, and sustainment. Formal quality improvement and change management techniques can elicit rapid and sustainable changes in trauma care. We provide a framework for change to increase compliance with fluid access in patients with major trauma.

\section{PROBLEM}

Adequate vascular access is required for the management of patients with trauma to provide a route for medications, resuscitative fluid, as well as intravenous contrast for diagnostic procedures. The importance of ensuring fluid access prior to patient deterioration should not be overlooked. Haemorrhage is still a major cause of trauma mortality and accounts for $31 \%$ of deaths in the first hour after injury. ${ }^{1}$ The American College of Surgeons' Advanced Trauma Life Support (ATLS) course mandates the insertion of two large-bore intravenous (IV) lines or the equivalent for patients with trauma. ${ }^{2-5}$

The University of Alberta Hospital is a level 1 trauma centre located in Edmonton, Alberta, that has a large geographical catchment area, diverse patient population and treats $>800$ of the 1500 major traumas within the Edmonton zone per year. As part of the quality improvement approach for the hospital's trauma programme, a review of care processes demonstrated inadequate vascular access in a significant portion of injured patients. An initial audit in January 2016 demonstrated that only $36.4 \%$ of patients with major trauma were receiving at least two $\geq 18$-gauge intravenous lines during the resuscitative phase of care (figure 1). A quality improvement plan was thus outlined to determine the root cause of poor compliance, with subsequent implementation of a change management process to resolve the gaps in care. While achieving two large-bore vascular access points is suggested in $100 \%$ of patients, non-modifiable factors (collapsed veins due to hypovolaemia, obese patients, small intravenous access already in place in the antecubital area) limit the potential success rate. One hundred per cent success rate was likely an unachievable goal in the hospital setting and risked reducing staff engagement during the early phases of change in this project. Therefore, our goal was to ensure that $\geq 90 \%$ of patients with trauma had at least two largebore vascular access points.

\section{BACKGROUND}

Ensuring large bore intravenous access during the resuscitative phase of a trauma patient's treatment for timely delivery of resuscitation fluids is required for definitive treatment. Poiseuille's law outlines that the flow rate through a cylinder is proportional to its radius to the power of four and inversely proportional to its length $\left(Q=\frac{\pi P r^{4}}{8 n l}\right)$. Therefore, the speed 
Adequate Vascular Access in Major Trauma Patients: January-November 2016

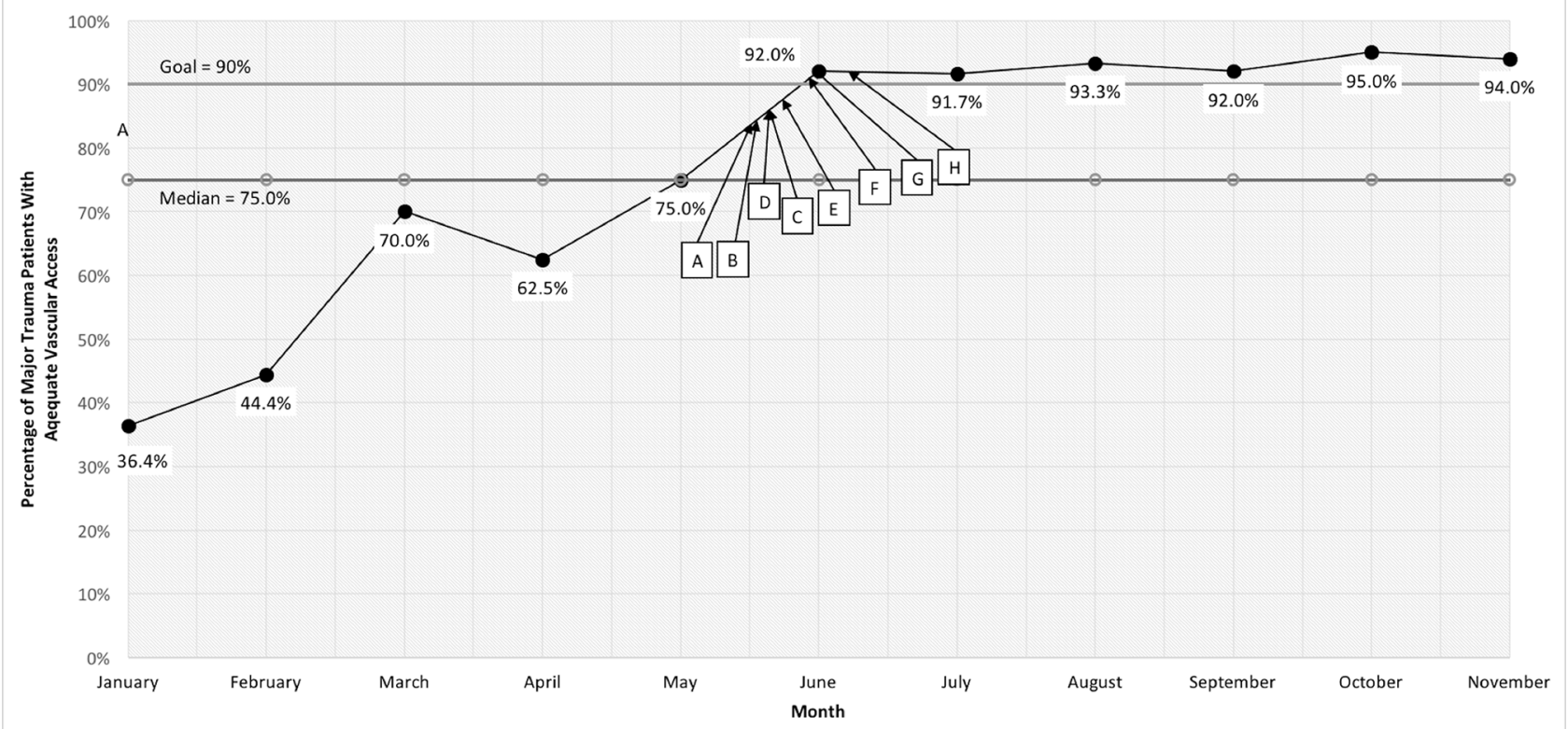

Figure 1 Run chart demonstrating the percentage of patients and trauma vascular access from January to November 2016.

of fluid through a catheter, and in turn the amount of fluid that can be infused per unit of time, is increased with shorter and larger radii tubes. Based on reported flow rates and only one intravenous, using an 18-gauge (instead of a 20 gauge) intravenous allows $1 \mathrm{~L}$ of fluid to be infused approximately 2 min and $2 \mathrm{~s}$ faster; a 16-gauge intravenous saves an additional 2 min and $4 \mathrm{~s}^{6}{ }^{6}$ Faster infusion of blood and blood products allows for rapid and quality resuscitation to avoid the pitfalls of haemorrhage. ${ }^{7}$ With advancement of damage control resuscitation, early adequate intravenous access allows life-saving treatment with plasma, platelets and blood in a timely fashion. ${ }^{78}$ Wider, shorter catheters facilitate re-establishment of a fluid balance that optimises trauma resuscitation and protocols recommend placement of 18-gauge or larger catheters for injured patients. ${ }^{3-5}$

To ensure these protocols were followed, we applied Deming and Donabedian quality improvement principles. Donabedian action involves two processes: performance monitoring to study current actions within the healthcare system and determine areas that require modification, and second, system design adjustment with resources to educate, train and create a structure that reduces patient risk. ${ }^{9}$ Deming's principles involve four crucial actions for change: planning the process to outline goals and defining metrics, changing the system, studying the effects of that change and acting to close the cycle and sustain change (also called plan, do, study and act, or PDSA rapid change cycles). ${ }^{1011}$

\section{METHODS}

\section{Measurement}

The study group initially performed a stakeholder analysis via multidisciplinary round-table discussion in January
2016 to determine which stakeholders were needed to define and support the project goals. The key stakeholders identified were: the hospital's trauma director, trauma manager, trauma coordinator, emergency department (ED) operational managers, ED clinical nurse educator, ED nursing staff, trauma team leaders, as well as emergency physicians.

Simultaneously, a 4-month chart audit (January-April 2016) was done on 54 patients with major trauma, using the Alberta Trauma Registry (ATR) to garner baseline data. The ATR collects data on patients with major trauma who have an injury severity score (ISS) $\geq 12$ and are $\geq 16$ years of age. During that time, there were 188 patients admitted with ISS $\geq 12$. Following April 2016, the trauma coordinator reviewed charts from all patients with trauma admitted, and further audits were done for patients likely to have an ISS $\geq 12$ based on their mechanism of injury and physiological parameters. From May-November 2016, we audited 142 of the 374 patients with trauma admitted to our hospital with an ISS $\geq 12$. Retrospectively cross-referencing audited charts with data from the ATR ensured that the included patients had an ISS $\geq 12$. For this study, we excluded patients with chronic or acute on chronic subdural haematomas.

Our baseline audits demonstrated that from January to April 2016, an average of $55.5 \%$ of the 54 patients with major trauma assessed had adequate fluid access during their resuscitative phase of care; this instilled us to implement change at the University of Alberta Hospital Emergency Department (UAH-ED) as a starting point for future system transformation.

A cause and effect analysis was completed within the UAH-ED from January to April 2016 to identify any gaps in 
care, garner staff support and to plan PDSA cycles. Based on stakeholder discussions, a cause-and-effect diagram and a quantitative Pareto diagram, were generated. Direct observation of work processes was performed, and a process map was created. Direct observation was done by the hospital's trauma coordinator, a member from the department of medicine quality management and a lead investigator of this study; these members directly observed care during several major trauma occurrences within the ED. Observation was directed towards identifying the allied health professionals involved, barriers to adequate intravenous access and potential changes that could be made to improve intravenous access rates. An antisolution technique, where stakeholders were asked to identify ways to make intravenous access rates worse, was applied to identify barriers to change and change cycles to address these barriers. A medical student conducted additional inperson qualitative interviews to evaluate attitudes, knowledge and barriers to successful compliance. Using input from the above, several PDSA cycles were developed and applied. These rapid change cycles focused on system changes, staff education and attitudes regarding intravenous access and took place over 2 months from May to June 2016, with ongoing audits continuing for another 6 months to assess sustainability.

The primary dependent variable for this study was the proportion of patients with adequate vascular access, defined as two of: $\geq 18$-gauge intravenous and/or intraosseous (IO) and/or central access with a $\geq 8$ French catheter placed during the resuscitation phase. Independent variables included all PDSA cycles, as we have described. Data were collected and grouped into a baseline data period from January to April 2016, a period of change from April to June 2016, and a sustainability follow-up period from June to November 2016. Data were collected and presented as averages for each month.

\section{Design}

Prior studies have examined the reasons for successful and sustained change in patient care. Evidence suggests that methods are successful when they involve specific solutions (for the site, personnel and problem) that are comprehensive, and engage multilevel stakeholders. ${ }^{12-16}$ We used these principles to develop an organised quality improvement initiative that focused on: baseline research to define the issue, stakeholder engagement to develop champions for change and specific (yet comprehensive) solutions for the issue and final implementation of the solutions. In this follow-up study, baseline data and stakeholder discussions helped support these principles and created an environment of trust and engagement that is crucial to achieving any change initiative success and future sustainability. ${ }^{11} 131416-18$

The PDSA cycles that were implemented from May to June 2016 are shown in table 1. One example is the reorganisation of the intravenous carts in the trauma bay, making large-bore intravenous more visible and accessible (figure 2). Other PDSA cycles focused on targeting and educating members of the trauma team regarding the importance and necessity of vascular access. Educational actions in May and June 2016 involved the trauma director presenting the importance of large-bore intravenous access to Trauma Team Leaders (TTLs), the trauma coordinator presenting the topic to all ED nurses at Wednesday morning educational huddles and one-to-one discussions between a lead investigator of this study and nurses working in the trauma bay. Further education was supported by an ED-wide email and memo to all nursing

Table 1 Rapid change cycles that were applied during the months of May-June

\begin{tabular}{|c|c|c|}
\hline $\begin{array}{l}\text { Letter on the run } \\
\text { chart (figure 1) }\end{array}$ & $\begin{array}{l}\text { Implementation } \\
\text { date }\end{array}$ & Improvement \\
\hline A & May 16 & $\begin{array}{l}\text { Reorganisation of intravenous carts. Separation of paediatric and adult intravenous. } \\
\text { Placed large-bore intravenous in visible and accessible locations and relabelled the } \\
\text { carts to enhance visibility. }\end{array}$ \\
\hline B & May 18 & Discussed the topic with groups of ED nurses at Wednesday morning huddles. \\
\hline B & May 18 & Educational posters \#1 and \#2 displayed. \\
\hline C & May $18-30$ & 1:1 discussions with ED nursing staff. \\
\hline D & May 19 & $\begin{array}{l}\text { Educational poster \#3 placed at nursing stations following feedback from frontline } \\
\text { staff. }\end{array}$ \\
\hline $\mathrm{E}$ & May 20 & $\begin{array}{l}\text { Conversations and written reminders to frontline staff and physician groups, } \\
\text { including staff and residents. }\end{array}$ \\
\hline $\mathrm{F}$ & May 30 & $\begin{array}{l}\text { Discussed at the trauma team leader (ED physician and surgeons) retreat by the } \\
\text { trauma director. }\end{array}$ \\
\hline G & May 31 & Second intravenous chart organised and labelled. \\
\hline $\mathrm{H}$ & June 6 & $\begin{array}{l}\text { Posters \#4 and \#6 were introduced and demonstrated educational tips and prompts } \\
\text { with regards to other options available if a large-bore intravenous cannot be } \\
\text { obtained, that is, cortice, } 10 \text { access. }\end{array}$ \\
\hline
\end{tabular}

ED, emergency department; IO, intraosseous. 


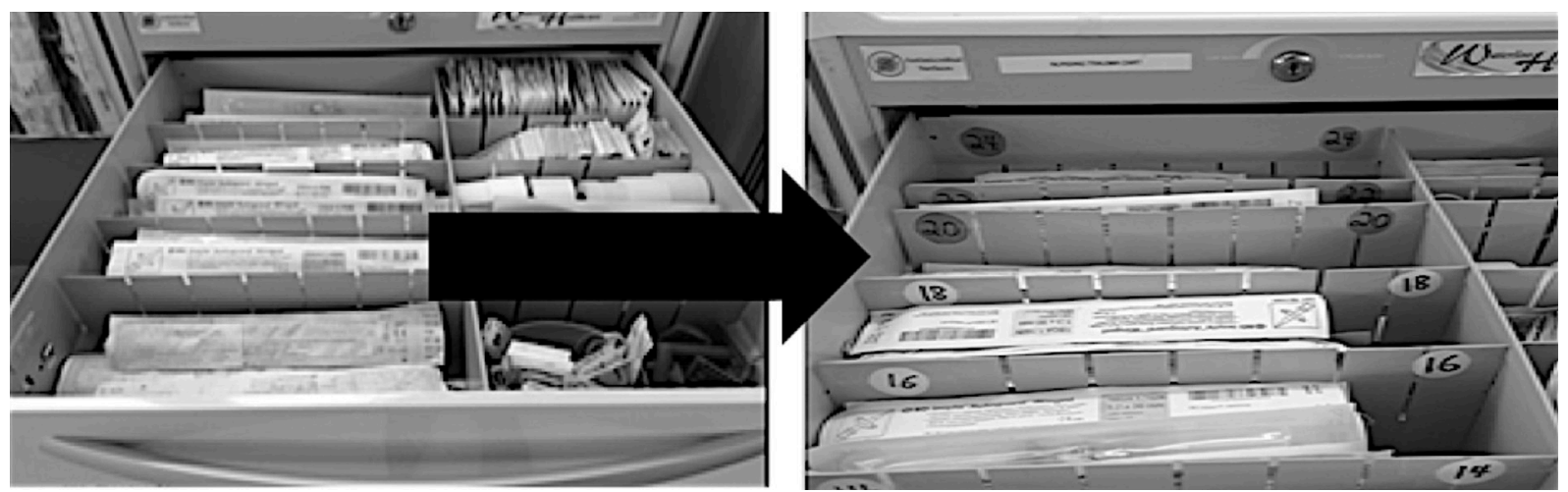

Figure 2 Intravenous cart drawer reorganisation and labelling changes. The image on the left is the intravenous cart prior to changes and the right is after changes. Changes included: optimisation of visibility of large-bore intravenous, separation of paediatric and adult intravenous and reorganisation of intravenous.

staff and physicians. Moreover, creative posters were continually posted within the ED to provide educational information, generate change and update staff working in the ED with audit results. Audit results were graphed and posted within the trauma bay and nursing stations each month to allow for transparency of results, feedback to staff and reflection.

\section{Strategy}

Baseline audits provided incentive to initiate a quality improvement project; they also demonstrated the Hawthorne effect, where vascular access increased from January to March (from $36.4 \%$ to $70.0 \%$ ) without any specific change interventions other than us openly auditing our processes to collect baseline data. However, the results were not sustainable, as the compliance rate dropped from $70 \%$ to $62.5 \%$ from March to April, likely due to the transient nature of Hawthorne effects. Transparency, sharing data with staff and promoting reflection help generate a culture that induces change internally. ${ }^{18} 19$

Once the specific problem was identified, we engaged our stakeholders to develop solutions. The core group of stakeholders included: nurses, ED physicians, surgeons, TTLs and residents and operational leadership in the ED and trauma. Engagement of stakeholders is crucial to the successful introduction, implementation and sustainability of any quality improvement initiative. ${ }^{11-13} 18$ Our multidisciplinary group discussions identified many barriers to proper care such as: poorly organised trauma intravenous carts, unclear protocols and lack of education regarding large-bore intravenous in patients with trauma that we organised to understand fully (figures 3 and 4). These barriers determined our change interventions for improvement (table 1). Without understanding the barriers to initiatives at the grass roots level, it is easy for one to be misled and make inappropriate assumptions

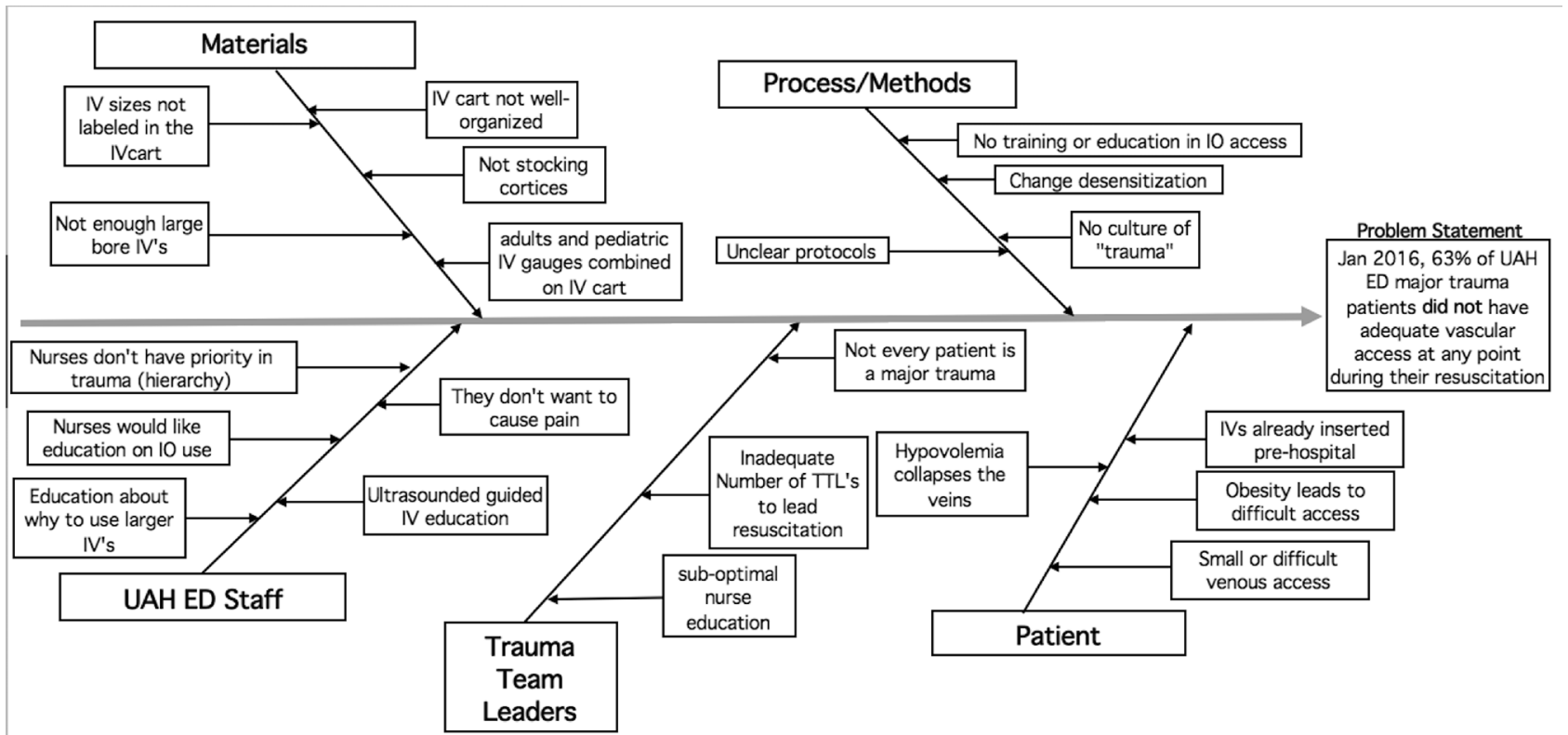

Figure 3 Fishbone diagram of the cause-and-effect analysis. IO, intraosseous; IV, intravenous; UAH ED, University of Alberta Hospital Emergency Department. 


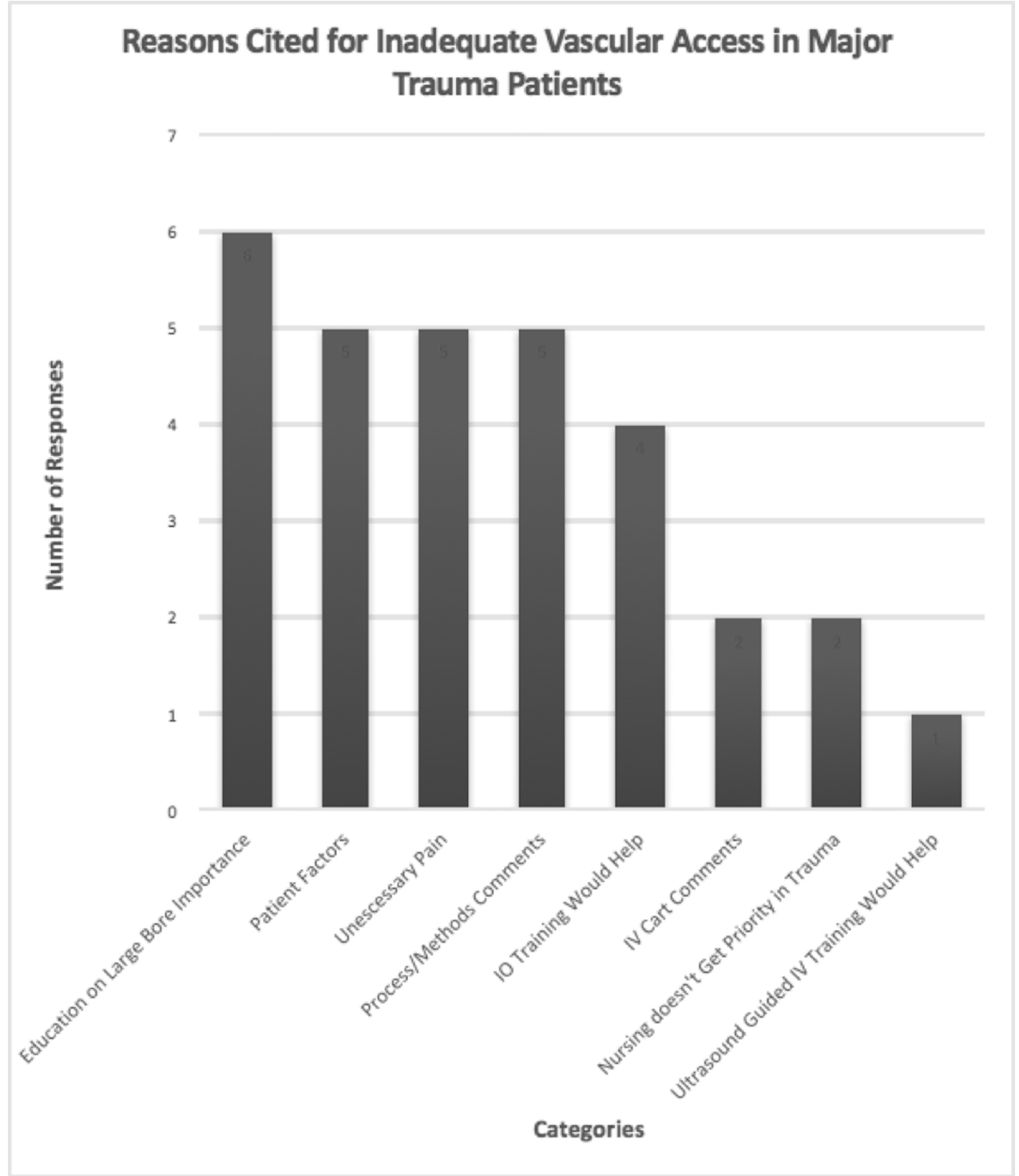

Figure 4 Pareto diagram display of the cause-and-effect analysis. IO, intraosseous; IV, intravenous.

around causes and potential solutions. Using participative techniques and endorsing feedback are efficacious methods for eliminating change resistance. ${ }^{12}{ }^{20}$ Similarly, engaging enthusiasts or energisers to lead change is an effective approach for healthcare changes. ${ }^{13}$ One-on-one discussions and ED staff presentations in May 2016 garnered these champions of change and resulted in the largest month over month intravenous compliance improvement (from $75 \%$ to $92 \%$ ). Others have recognised these advocates as key to increasing compliance with existing protocols. ${ }^{17} 1821$ Therefore, discussions with staff identified areas for change management and quality improvement, while simultaneously generating awareness, acceptance, trust and involvement for any upcoming changes.

\section{RESULTS}

The baseline data audit involved 54 patients with major trauma, over the 4-month time frame for the baseline audit, only $55.5 \%$ of major trauma patients received adequate vascular access. Change cycles were initiated, and audits were performed simultaneously. Audits in May and June identified 24 and 25 cases and a compliance rate of $75 \%$ and $92 \%$, respectively. Throughout the study period (January-November 2016) 196 patients with trauma were included. Compliance with adequate intravascular access $>90 \%$ was sustained from June to the end of November 2016 (figure 1).

Stakeholder analysis identified appropriate staff groups to engage, which established key members for our multidisciplinary group discussions. Multidisciplinary group discussions identified various barriers to attaining adequate vascular access. These factors were then explicitly mapped out in a cause-and-effect Fishbone diagram (figure 3). A Pareto diagram was then created to identify the $20 \%$ of factors that were causing $80 \%$ of the problem (figure 4). The most significant alterable impediments that we identified were protocol uncertainty and lack of 


\begin{tabular}{|c|c|}
\hline $\begin{array}{r}\text { WHAT DO WE HA } \\
\square \square \square \square \square \square \square \\
\text { To infius }\end{array}$ & 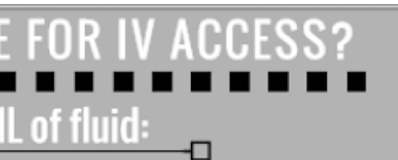 \\
\hline An 18 vs 20 & A 16 vs 20 \\
\hline gauge IV & gauge IV \\
\hline saves $2: 23$ & saves $4: 47$ \\
\hline What does th & time mean! \\
\hline $\begin{array}{l}\text { - Burn } 200 \text { calories } \\
\text { by sprinting } \\
\text { - Gordon Ramsey } \\
\text { cooks a rare steak }\end{array}$ & $\begin{array}{l}\text { - Make it } 1 / 10 \text { th of } \\
\text { the way through the } \\
\text { Tim-Horton's line } \\
\text { - Gas up you car }\end{array}$ \\
\hline More importantly: & More importantly: \\
\hline $\begin{array}{l}\text { - Airway can be } \\
\text { managed } \\
\text { - } 1 \text { extra unit of } \\
\text { blood can be given } \\
\text { with an } 18 \text { gauge IV }\end{array}$ & $\begin{array}{l}\text { - Surgeons could } \\
\text { open the abdomen } \\
\text { - A chest tube can be } \\
\text { inserted }\end{array}$ \\
\hline
\end{tabular}

Figure 5 Educational poster used in the emergency department. IV, intravenous.

education regarding adequate vascular access in patients with trauma.

\section{DISCUSSION}

Leadership and programme support achieved during the baseline data collection and root cause analysis set the stage for successful change management. Leadership can help prioritise quality improvement projects, remove barriers and provide additional supports as needed. ${ }^{17} 1821$ Discussions were held to share the background data and status with staff by both management and leadership alike, and the collaborative solution was presented with a clear protocol designed by frontline staff but supported by key organisational leaders.

Education regarding flow rates as they relate to timely fluid resuscitation and implications of inadequate fluid access were also discussed. These efforts were strengthened with weekly educational huddles and cueing posters to engage internal staff discussion and action (figure 5). Introducing these comprehensive and problem-specific solutions in rapid succession resulted in protocol compliance increasing from $62.5 \%$ to $92 \%$ from April to June 2016 (figure 1). We suggest that the innovative visual posters and messaging, as compared with memos and conventional hospital announcements, which represent organisational hierarchy, supported continued discussion and engagement within the team. By introducing creativity, and flattening the hierarchy, the results demonstrated ongoing improvements from April to June and were immediately sustained in the months following our success. One way this was done was through clinical vignettes where near misses related to the need for intravenous access (eg, a non-impressive trauma that had a hidden carotid injury that bled extensively) were explained to staff to help engage and induce change. Frequently updating the ED with progressively more informative posters was successful to retain engagement and promote a sustainable culture change, as our results were maintained beyond June 2016.

To ensure sustainability of the change that we have achieved, adequate vascular access will be continuously audited as part of the standard University of Alberta Trauma Quality Improvement programme. Lastly, recognising that many of the nursing staff in our ED are not trained to perform IO procedures, we plan to increase such training opportunities. Additionally, we are currently looking at large-bore intravenous compliance at our other trauma sites within the zone (city) as well as provincially. This study is being used as a toolkit, along with the ability to connect with quality consultants, to help spread the success of our project.

\section{Lessons and limitations}

Despite the ongoing success of our project, this report is limited by a sustainability follow-up period of 6 months. Typically, it is recommended to assess for sustainability at 8 months and beyond. Additionally, it is difficult to assess the replicability of this process; applying it to a broader system by replicating the process in a similar hospital or throughout the trauma system, that is, prehospital services, would allow a greater understanding of potential barriers that diverse providers of care face.

Despite increasing compliance with ATLS accepted protocols, our study did not evaluate health outcomes in our patient population. Future studies are suggested to determine the clinical correlation of increasing intravenous access in patients with major trauma. We did consider adverse events, such as intravenous-related infections, thrombophlebitis, interstitial intravenous and pain, as potential balancing measures for this project. Methods for reducing complications from intravenous access, such as the use of bundles to reduce infection, have been outlined and could be applied if we recognised unwanted complications from our project. ${ }^{22}{ }^{23}$ However, these balancing measures were not adequately documented, and we could not effectively determine the rates before or after the intervention. Ensuring thorough documentation of fluid resuscitation related-volumes, complications and intravenous access attempts would allow a better understanding of some balancing measures to ensure that there were no negative outcomes resulting from this quality improvement initiative. 


\section{CONCLUSION}

We achieved successful compliance with a protocol that outlines appropriate fluid access in patients with major trauma at the UAH. Our systematic quality improvement method hinged on Donabedian and Deming principles that involved a thorough background data and stakeholder analysis prior to change management and facilitated rapid and positive uptake of change. This allowed us to achieve our quality improvement goal and sustain for a period of 6 months. We suggest that engagement of staff, use of quality tools, as well as timely and transparent data collection can lead to success with quality improvement initiatives.

Acknowledgements We would like thank our colleagues from the Emergency Department at the University of Alberta, Alberta Trauma Services, and others who willingly provided important feedback, insight and expertise that greatly assisted the research and success of this project.

Contributors KV implemented change processes and drafted and edited the paper. RS edited the paper and helped implement change processes. PM revised the drafted paper, consulted for change management approaches and provided quality improvement tools for the project. BT and VF edited the drafted paper and consulted with development of the draft paper. SW initiated the collaborative project, change management actions and edited the draft paper.

Competing interests None declared.

Ethics approval Ethics was submitted and approved by the Research and Ethics Board at the University of Alberta.

Provenance and peer review Not commissioned; externally peer reviewed.

Open Access This is an Open Access article distributed in accordance with the Creative Commons Attribution Non Commercial (CC BY-NC 4.0) license, which permits others to distribute, remix, adapt, build upon this work non-commercially, and license their derivative works on different terms, provided the original work is properly cited and the use is non-commercial. See: http://creativecommons.org/ licenses/by-nc/4.0/

(C) Published by the BMJ Publishing Group Limited. For permission to use (where not already granted under a licence) please go to http://www.bmj.com/company/ products-services/rights-and-licensing/

\section{REFERENCES}

1. Sobrino J, Shafi S. Timing and causes of death after injuries. Proc 2013;26:120-3.

2. The alberta college of paramedics. Alberta Occupational Competency Profile (AOCP) Upgrade "Gap" Training Program. In. Emergency Medical Technician (EMT) Intravenous Therapy Module.
3. National Institute for Health and Care Excellence. Major trauma: assessment and initial management: National Clinical Guideline Center, 2016.

4. Cotton BA, Jerome R, Collier BR, et al. Guidelines for prehospital fluid resuscitation in the injured patient. $J$ Trauma 2009;67:389-402.

5. American College of Surgeons Committee on Trauma. ATLS student course manual: advanced trauma life support. 9th edn, 2012.

6. Barcelona SL, Vilich F, Coté CJ. A comparison of flow rates and warming capabilities of the Level 1 and rapid infusion system with various-size intravenous catheters. Anesth Analg 2003;97:358-63.

7. Engels PT, Passos E, Beckett AN, et al. IV access in bleeding trauma patients: a performance review. Injury 2014;45:77-82.

8. Dzik WH, Blajchman MA, Fergusson D, et al. Clinical review: canadian national advisory committee on blood and blood productsmassive transfusion consensus conference 2011: report of the panel. Crit Care 2011;15:242.

9. Block DJ. Quality Improvement in Health Care - Donabedian's Principles of Quality Improvement. Chapman T, ed. Healthcare outcome management: strategies for planning and evaluation. 1. sudbury, MA: Jones and Bartlet, $2006: 9-24$.

10. The W. Edwards Deming Institute. The Plan, Do, Study, Act Cycle. https://www.deming.org/theman/theories/pdsacycle

11. van Tiel FH, Elenbaas TW, Voskuilen BM, et al. Plan-do-studyact cycles as an instrument for improvement of compliance with infection control measures in care of patients after cardiothoracic surgery. J Hosp Infect 2006;62:64-70.

12. Grol R, Grimshaw J. From best evidence to best practice: effective implementation of change in patients' care. Lancet 2003;362:1225-30.

13. Mann D. The missing link: lean leadership. Front Health Serv Manage 2009;26:15-26.

14. Mayer JA, Dubbert PM, Miller M, et al. Increasing handwashing in an intensive care unit. Infect Control 1986;7:259-62.

15. Grol R. Successes and failures in the implementation of evidencebased guidelines for clinical practice. Med Care 2001;39:II-46.

16. Grimshaw JM, Shirran L, Thomas R, et al. Changing provider behavior: an overview of systematic reviews of interventions. Med Care 2001;39:2-45.

17. Coffey M, Cornish P, Koonthanam T, et al. Implementation of admission medication reconciliation at two academic health sciences centres: challenges and success factors. Healthc Q 2009;12 Spec No Patient:102-9.

18. Berwick DM, Feeley D, Loehrer S. Change from the inside out: health care leaders taking the helm. JAMA 2015;313:1707-8.

19. Iedema R, Ball C, Daly B, et al. Design and trial of a new ambulanceto-emergency department handover protocol: 'IMIST-AMBO'. BMJ Qual Saf 2012;21:627-33.

20. Waddell D, Sohal AS. Resistance: a constructive tool for change management. Management Decis 1998;36:543-8.

21. Fernandes $O$, Shojania KG. Medication reconciliation in the hospital. Healthc Q 2012;15:42-9.

22. Youn SH, Lee JC, Kim Y, et al. Central venous catheter-related infection in severe trauma patients. World J Surg 2015;39:2400-6.

23. Pronovost PJ, Goeschel CA, Colantuoni E, et al. Sustaining reductions in catheter related bloodstream infections in Michigan intensive care units: observational study. BMJ 2010;340:c309. 